\section{Interaction of apolipoprotein-E with ATP-binding cassette transporters: Impact on neuronal cholesterol homeostasis and amyloid-beta peptide generation}

\section{B Garner}

Prince of Wales Medical Research Institute, Randwick, New South Wales, Australia

Maintenance of an adequate supply of cholesterol is important for neuronal function, whereas excess cholesterol promotes amyloid precursor protein (APP) cleavage generating toxic amyloid-beta (A-beta) peptides. To gain insights into the pathways that regulate neuronal cholesterol level, we investigated the potential for reconstituted apolipoprotein-E (apoE) discs, resembling nascent lipoprotein complexes in the central nervous system, to stimulate neuronal $[3 \mathrm{H}]$-cholesterol efflux. The apoE discs potently accelerated cholesterol efflux from primary human neurons and cell lines. The process was saturable (17.5 $\mu \mathrm{g} \mathrm{apoE} / \mathrm{ml})$ and was not influenced by APOE genotype. HPLC analysis of cholesterol and cholesterol metabolites effluxed from neurons indicated that $<25 \%$ of the released cholesterol was modified to polar products (eg 24-hydroxycholesterol) that diffuse from neuronal membranes. Thus, most cholesterol $(\sim 75 \%)$ appeared to be effluxed from neurons in a native state by means of a transporter pathway. ATP-binding cassette transporters ABCA1, $\mathrm{ABCA} 2$ and $\mathrm{ABCG} 1$ were detected in neurons and neuroblastoma cell lines and transient and stable expression of these complementary DNAs showed that ABCG1 preferentially stimulated cholesterol efflux to apoE discs. In addition, ABCG1 expression in $\mathrm{CHO}$ cells that stably express human APP resulted in $\sim 60 \%$ reduction in A-beta generation. ABCA1 also stimulated cholesterol efflux to apoE discs and inhibited A-beta generation, although both to a lesser degree than ABCG1, whereas ABCA2 did not modulate either of these processes. These data indicate that ABCG1 and ABCA1 play a significant role in the regulation of neuronal cholesterol efflux to apoE discs and in suppression of APP processing to generate A-beta peptides.

\section{The role of apolipoprotein $E$ in the pathology of schizophrenia and bipolar disorder}

\section{B Dean, A Digney, E Thomas, E Scarr}

The Rebecca L. Cooper Research Laboratories, The Mental Health Research Institute, Victoria, Australia

It is now widely accepted that the changes in the molecular milieu of the human central nervous system (CNS) that precipitate frank psychiatric illness is because of the impact of susceptibility genes and as yet to be identified environmental stressors (Tsuang Biol Psych 2000, 47 210). Therefore, the demonstration of altered levels of apolipoprotein E (Dean et al. Bio Psych 2003, 54 616-622; Digney et al. Biol Psych 2005, 57 711-715) in the CNS from subjects with schizophrenia and bipolar 1 disorder suggested that this important glial-derived protein could be involved in the pathology of these disorders. Moreover, treating rats with haloperidol decreases frontal cortical apolipoprotein E (Dean et al. Bio Psych 2003, 54 616-622) suggesting that regulating apolipoprotein E might be a mechanism by which antipsychotic drugs could achieve therapeutic outcomes. Our data on haloperidol, which presumably affects apolipoprotein E levels by blocking dopaminergic activity, also added to studies showing that neurotransmitters such as serotonin, acetylcholine and glutamate (Deecher et al. J Neurosci Res 1993, 35 246-256; Andre et al. Euro J Neurosci 1994, 6 1702-1709; Gallo et al. J Neurochem 1987, 14 1801-1809) (all important in the pathology of psychiatric disease (Dean PMJ 2002, 78 142-148; World J Biol Psychaitr 2002, 3 125-132) can affect astrocytic function and thus apoE expression. Combining studies on apolipoprotein $\mathrm{E}$ with many of studies showing changes in neuronal-derived proteins in schizophrenia and bipolar disorder (Dean Postgrad Med J 2002, 78 142-148, World J Biol Psychaitr 2002,3 125-132), it can be proposed that changes in tripartite synaptic function, the matrix involving pre- and postsynaptic neurons and associated glia, are altered in the CNS of some subjects with psychiatric illnesses. Evidence to support this hypothesis will be reviewed and potential mechanism of action and symptom outcomes will be discussed. 